\title{
EARLY DIAGNOSIS OF TUBERCULOUS PERITONITIS BY NESTED PCR AND AUTOMATED CULTURE TECHNIQUE
}

\author{
Apurba Sankar Sastry, Sandhya Bhat K, Anand Sankar Sastry, Kumudavathi M S, \\ 1. Assistant Professor, MD, DNB, Department of Microbiology, Meenakshi Medical College, Kanchipuram, \\ Tamilnadu. \\ 2. Assistant Professor, MD, DNB, Department of Microbiology, Meenakshi Medical College, Kanchipuram, \\ Tamilnadu \\ 3. Senior Resident, MD, Department of Medicine, Karpaga Vinayaga Medical College, Kanchipuram,Tamilnadu. \\ 4. Tutor, D (Bact), Department of Microbiology, Meenakshi Medical College, Kanchipuram, Tamilnadu.
}

\section{CORRESPONDING AUTHOR}

Dr. Apurba Sankar Sastry, Department of Microbiology,

Meenakshi Medical College and Research Institute,

Kanchipuram, Near Chennai, India

E-mail: drapurbasastry@gmail.com

Ph: 00919444327314.

ABSTRACT: INTRODUCTION: Early laboratory diagnosis of tuberculous peritonitis (TBP) is crucial to start antitubercular chemotherapy and to prevent its complications. However conventional methods are either less sensitive or time consuming. Hence the diagnostic potential of BacT/ALERT and polymerase chain reaction (PCR) was evaluated in this study. MATERIAL AND METHOD: The study group comprised of 21 cases and nine controls. The cases were divided into confirmed sub group (seven cases) - smear or culture or histopathologically proven and probable subgroup (fourteen cases) - clinically suspected cases. Ziehl Neelsen (ZN), Auramine Phenol (AP) staining, Lowenstein Jensen (LJ) culture, BacT/ALERT and nested Polymerase chain reaction (PCR) targeting IS6110 were carried out on all the patients. RESULTS: Sensitivity of ZN, AP staining and LJ culture were found to be $23.8 \%$, $28.5 \%$ and $57.1 \%$ respectively. Whereas BacT/ALERT and nested PCR showed a sensitivity of $76.1 \%$ and $90.4 \%$ respectively. The mean detection time of growth by LJ culture was 32.10 days where as that of BacT/ALERT was 21.28 days. The contamination rates in LJ culture and BacT/ALERT were $5.0 \% \%$ and $10.0 \%$ respectively. CONCLUSION: Nested PCR and BacT/ALERT found to be more sensitive compared to LJ culture and smear microscopy. BacT/ALERT detects mycobacterial growth at a faster rate with less contamination rate compared to LJ culture. As both false negative and false positive results are reported on nested PCR, so alone it should not be used as a criterion for initiating or terminating the therapy but should be supported by clinical, radiological, cytological and other microbiological finding.

KEY WORDS - Tuberculous peritonitis, nested PCR, BacT/ALERT

INTRODUCTION: Tuberculous peritonitis (TBP) accounts for 10-12 \% of all cases of extrapulmonary tuberculosis $(1)$. Although it is uncommon in western world, it remains a serious problem in Asia especially in HIV positive patients. Laboratory methods play a crucial role in establishing the diagnosis, early starting and monitoring the chemotherapy, preventing the transmission, identifying the changing pattern of epidemiology and detection of resistance to drug. Diagnosis of TBP is often difficult due to paucibacillary nature of the disease. Though ZN staining is rapid and cheap but it is neither sensitive nor specific requiring a minimum bacillary load of $10^{4} / \mathrm{ml}^{(2)}$. Culture is more sensitive than ZN staining with detection limit of $10-100$ 
bacilli/ml but requires prolonged incubation time of 6-8 weeks because of the long generation time of tubercle bacilli(3). Even histology is also at times not conclusive.

Many newer diagnostic modalities like automated microbial detecting systems and molecular methods have come up with increase isolation rate and early detection of the organism. BacT/ALERT Microbial Detecting System is based upon colorimetric detection of $\mathrm{pH}$ change which occurs due to $\mathrm{CO}_{2}$ production by Mycobacteria (3). Not much studies are there evaluating diagnostic potential of BacT/ALERT for tuberculosis (TB) especially for TBP. Polymerase chain reaction (PCR) is another alternate for the early diagnosis of TBP. IS6110 is a transposone present as multiple repetitive elements in the genome of M. tuberculosis complex with variable copy numbers ranging from 0 to 26 (3). Due its repetitive nature, tests using IS6110 as primer yield higher sensitivity. Nested polymerase chain reaction (PCR) using IS6110 though has been widely evaluated in the diagnosis of TB but fewer studies are only available for the diagnosis of TBP. Hence we have evaluated the potential of BacT/ALERT and nested PCR for the diagnosis of TBP.

MATERIAL AND METHODS: The present study was carried out in the department of Microbiology of a tertiary care centre and was approved by the institute ethical committee.

CASES: Samples were collected from 21 cases of TBP admitted to the hospital, during July 2010 to September 2012 and divided to the following subgroups.

a) Confirmed TBP subgroup: comprised of seven case confirmed by smear, culture or histopathology.

b) Probable TBP subgroup: comprised of fourteen clinically suspected cases having features like pyrexia of unknown origin, abdominal tenderness, weight loss, night sweat and / or positive Mantoux test and / or elevated ESR and / or positive peritoneal fluid cytology like pleocytosis, elevated protein level and reduced sugar level and / or evidence of pulmonary or any other organ tuberculosis and patient improving on antitubercular drug (ATT) (Table 1).

CONTROLS: Nine peritoneal fluids were collected from known cases of bacterial peritonitis. Approximately $2 \mathrm{ml}$ of sample was collected aseptically. One $\mathrm{ml}$ of the sample was used for both smear and culture, $0.5 \mathrm{ml}$ was used each for BacT/ALERT \& PCR. Smears were made from the samples and were stained by both Ziehl-Neelsen technique and auramine phenol method as per the standard methods (3). Cultures were carried out on Lowenstein - Jensen (L-J) egg based medium as per standard method (4).

BACT/ALERT: The processing of peritoneal fluid was done as per as the manufacturer's instruction (5). Inoculated BacT/ALERT MP bottles were loaded in MB/BacT instrument for the incubation and the growth was monitored. BacT/ALERT MP bottle with mycobacterial growth produced a color change of sensor from dark green to yellow, changing the screen color to yellow. The positive BacT/ALERT MP bottles were unloaded, vortexed heavily to make the large clumps to break and suspend uniformly. The mycobacterial growth was confirmed by performing ZN staining from it.

POLYMERASE CHAIN REACTION: The PCR was carried out as per the method described elsewhere (6). The peritoneal fluid was centrifuged at $3000 \mathrm{rpm}$ for 15 minutes, and then the supernatant was discarded. To the sediment, $50 \mu \mathrm{l}$ lysozyme was added followed by incubation at $37^{\circ} \mathrm{C}$ in water bath overnight. $70 \mu \mathrm{l} 14 \%$ SDS and $6 \mu \mathrm{l}$ proteinase $\mathrm{K}$ were added and the 
mixture was incubated at $65^{\circ} \mathrm{C}$ for 15 minutes. Subsequently, $10 \mu \mathrm{l}$ of $5 \mathrm{M} \mathrm{NaCl}$ and $80 \mu \mathrm{l} \mathrm{of} 10 \%$ CTAB (activated at $55^{\circ} \mathrm{C}$ ) were added and the final mixture was incubated at $65^{\circ} \mathrm{C}$ for 10 minutes. Then, $800 \mu \mathrm{l}$ of phenol, chloroform and iso amyl alcohol was added in ratio of 25: 24: 1 . The mixture was spun at $10000 \mathrm{RPM}$ for 10 minutes at $4^{\circ} \mathrm{C}$. Supernatant was collected, $600 \mu \mathrm{l}$ of ice cold isopropanol was added and was incubated at $-20^{\circ} \mathrm{C}$ overnight. Next day it was spun for 10 minutes at $12000 \mathrm{RPM}$ at $4^{\circ} \mathrm{C}$. After the fluid was drained out, it was kept in the incubator for drying. Finally, $20 \mu \mathrm{l}$ Tris buffer was added and it was stored at $-20^{\circ} \mathrm{C}$.

Primers were validated by blasting the primer sequence used for detection of M.tuberculosis in the genome database of all the organisms in the web site ( http://www.ncbi.nlm.nih.gov/blast/) and were found to be specific for the organism. The sequence of the TB PCR Primers were: first set conventional PCR round-forward primer (FL): 5' CTC AAG TGA AGG AGG CAA CC - 3' and reverse primer (FR): 5' TGG GCT AGG GTG TTG ATC TC - 3' where as for the nested PCR: forward primer (NFL): 5' CGT CTG GAG CGT GAC CTA CT - 3' and reverse primer (NFR): 5' GAC ATC TCG ACG GTC AGT CA - 3' respectively.

PCR mix consisted of $4 \mu \mathrm{l}$ of extracted DNA, $10 \mu \mathrm{l}$ of $2 \mathrm{X}$ readymade master mix, $2 \mu \mathrm{l}$ of $10 \mu \mathrm{M}$ Primer (FL and FR for $1^{\text {st }}$ round PCR and NFL and NFR for nested PCR) and $10 \mu \mathrm{l}$ of milliQ water. The settings of the thermocycler programmed were similar for both first round and nested PCR except for the primers used. Total 30 cycles were carried out in each round, each cycle comprised of initial denaturation at $94^{\circ} \mathrm{C}$ for 5 minutes, denaturation occurred at $94^{\circ} \mathrm{C}$ for $60 \mathrm{~s}$, annealing occurred at $56^{\circ} \mathrm{C}$ for $60 \mathrm{~s}$, amplification occurred at $72{ }^{\circ} \mathrm{C}$ for $60 \mathrm{~s}$ followed by final extension at $72{ }^{\circ} \mathrm{C}$ for $7 \mathrm{~min}$. Agar gel electrophoresis was carried out and bands are visualized under UV rays. The MTB specific nested PCR product size is $219 \mathrm{bp}$ length. The bands were separated in the agarose gel according to the molecular weight which then checked by comparing the bands with standard molecular weight marker.

RESULT: The microbiological findings of various tests were depicted in table 1 . ZN staining and AP staining have shown $23.8 \%$ ( 5 out of 21 ) and $28.5 \%$ ( 6 out of 21 ) sensitivity respectively whereas LJ culture was found to be $57.1 \%$ sensitive (12 out of 21 cases). BacT/ALERT microbial detecting system detected sixteen out of 21 cases (76.1\%). There was one case (in probable group) which was detected by BacT/ALERT but was negative by nested PCR. The contamination rate of BacT/ALERT and LJ culture in the present study was found to be $5.0 \%$ and $10.0 \%$ respectively. Mean detection time of BacT/ALERT in all the isolates was 21.28 days compared to 32.10 days taken by LJ culture. Gel image after nested PCR targeting IS6110 of M.tuberculosis was shown in figure 1. Nested PCR could detect nineteen out of 21 (90.4\%) cases of TBP where as one out of nine $(11.1 \%)$ in control group was also detected positive by nested PCR. There were four cases detected by nested PCR but was negative by BacT/ALERT. Both the cases and controls were age and gender matched.

DISCUSSION: Tuberculous peritonitis (TBP) is the sixth most frequent site of extrapulmonary involvement ${ }^{(7)}$. Although an infrequent disease, TBP with its non-specific symptoms and sinister clinical course can be easily confused with other intraabdominal diseases. Both the incidence and severity of TBP are expected to increase with increasing incidence of HIV infection. Hence, early and prompt diagnosis of the condition contributes to early start of anti tubercular therapy, thus preventing thereby the complications due to the condition. However, accurate laboratory diagnosis of TBP still continues to be a challenge.

Journal of Evolution of Medical and Dental Sciences/Volume1/Issue5/November-2012Page-756 
In the present study, acid fast microscopy by ZN and AP staining showed low sensitivity which can be explained because of the paucibacillary nature of peritoneal fluid. A high bacterial load $\left(>10^{4}-10^{5}\right.$ bacilli / $\left.\mathrm{ml}\right)$ is needed in the specimen to render an AFB microscopy result positive(2). Various studies documented similar sensitivities of smear microscopy for TBP of < $20 \%{ }^{(6)}$. AP staining is found to be more sensitive than ZN staining as the smears can be examined by AP staining at 250x or 450x, covering larger area in the same time (12).

Culture by LJ media showed an overall sensitivity of $57.1 \%$. Similar sensitivities had been documented in different studies ranging from $20-75 \%{ }^{(6)}$. Low sensitivity of LJ culture could be due paucibacillary nature of the peritoneal fluid as minimum detection threshold being 100 1000 bacilli per $\mathrm{ml}$ (3). Other reasons could be due to presence of dead bacilli, intake of other broad spectrum antibiotics inhibiting Mycobacteria and prior starting of ATT.

The BacT/ALERT microbial detecting system showed an overall sensitivity higher than the LJ culture and specificity of $100 \%$. In confirmed \& probable sub group the sensitivity was $100 \%$ and $64.2 \%$ respectively. Out of sixteen mycobacterial isolates were recovered in our study, all (100\%) were recovered by BacT/ALERT compared to $75.0 \%$ (twelve isolates) recovery rate of $L J$ culture. Other studies also have shown recovery rate of $91 \%$ to $94 \%$ by BacT/ALERT (7,8). The contamination rate of BacT/ALERT and LJ culture in our study was found to be $5.0 \%$ and $10.0 \%$ respectively. Similar reports were documented in different studies(7). Mean detection time of BacT/ALERT in all seven isolates was 21.28 days compared to 32.10 days taken by LJ. The mean detection time of BacT/ALERT in smear positive specimen was 17 days where as that in smear negative specimens were 22.8 days. Various studies documented mean detection time of 15-16 days by BacT/ALERT (7-8). Early detection of the bacilli in these studies could be due to inclusion of both pulmonary and extrapulmonary specimens in these studies.

In the present study, nested PCR showed an overall sensitivity of $90.4 \%$ which was higher than culture but differed in the confirmed (100\%) and probable (85.7\%) subgroup. In an European study by T.zoanopoulos et al, all the three suspected cases of TBP were found positive by PCR targeting IS6110 (9). As the sample size was very less and response to ATT was considered as final inclusion criterion for TBP, so the sensitivity was found higher in this study which might be misleading.

In our study, there were two cases which could not be detected by nested PCR (false negative results). The reasons of lower sensitivity might be due to many reasons: First, presence of PCR inhibitors which are found to be more associated with extrapulmonary specimen compared to pulmonary specimen (10-11). A better extraction procedure like immunomagnetic separation technique should be used which could capture all M.tuberculosis DNA, but not inhibitors (10). Various resin matrixes like 'Gene releaser preparations' which absorb inhibitors without entailing further loss of DNA may also be used (10). Second, poor lysis of Mycobacteria in the extraction procedure due to the complexity of the cell wall $(9,12)$. Third, some strains of M.tuberculosis in Asia lack the IS6110 sequence (13-15). Hence few cases might have got undetected if Mycobacteria present in the samples lack the IS6110 sequence Fourth, low number of bacilli present in the peritoneal fluid.

The overall specificity of nested PCR in our study was $88.8 \%$. There was one out of nine controls which was detected positive (false positive) by our study where actually Escherichia coli was isolated and patient responded to the antibiotics. The reasons of false positive results could be due to cross contamination during initial handling or due to amplicon carry over 
contamination which can be overcome by using a single tube nested PCR (10). It is also found that use of dUTP/Uracil-N-glycosylase could decrease the amplicon contamination(10).

There were seven cases which were detected by nested PCR but were rendered negative by LJ culture. The gain in the sensitivity $(40 \%)$ over the so called "gold standard" can be explained by low detection limit of nested PCR of as few as 10 bacilli per $\mathrm{ml}$. The sensitivity gain in the etiological diagnosis by nested PCR substantiates the diagnostic utility of this method. Moreover nested PCR offers the advantages like speed in obtaining the result, option of referring the sample rather than the patient to a specialized centre.

There was one case (in probable group) which was detected by BacT/ALERT but was negative by nested PCR whereas, there were four cases which was detected by nested PCR but was negative by BacT/ALERT. So the combined sensitivity of BacT/AERT and nested PCR in the probable group (92.8\%, 13 out of 14 cases) is more than individual sensitivity of BacT/AERT (64.2\%) and nested PCR(85.7\%).

In conclusion, diagnosis of TBP is often difficult due to the atypical clinical presentation and paucibacillary nature of the sample. Both Nested PCR and BacT/ALERT found to be more sensitive compared to conventional LJ culture and smear microscopy. BacT/ALERT takes lesser time to detect growth and associated with less contamination rate compared to conventional LJ culture. We suggest that nested PCR should deserve a place in laboratory diagnosis of TBP but careful adherence to the test protocol is mandatory. As both false negative and false positive results are reported on PCR, hence PCR alone should not be used as a criterion for initiating or terminating the therapy. This should be supported by clinical, radiological, cytological and other microbiological finding (smear microscopy, culture by conventional and automated system) to guide the clinician in decision making for appropriate therapy whenever possible.

The limitations of the present study are as follows. 1) It was based on clinical criteria which though had been validated well before but cannot be considered as gold standard. 2) Internal controls were not used for nested PCR. We believe that use of internal control could have helped in eliminating the possibility of PCR inhibitors. 3) We have not evaluated the diagnostic potential of these tests in HIV positive patients, where the sensitivity would have been more. 4) Only IS6110 is targeted in our study which might be absent in some Indian isolates of M.tuberculosis. 5) The sample size is small. Since TBP is relatively rare, it is difficult to get more patients. If the sample size can be increased then it will provide a clear picture about the parameters.

\section{REFERENCES:}

1. Basu S, Ganguly S, Chandra PK, Basu S. Clinical profile and outcome of abdominal tuberculosis in Indian children. Singapore Med J 2007 Oct;48(10):900-5.

2. Survashi B.S, Bhanu V, Seth P, Suresh N, Arora J, Rana T. Utility of polymerase chain reaction in diagnosis of tuberculosis from samples of bone marrow aspirate. Am J Trop Med Hyg 2006;75(5):960-3.

3. Murray PR. Manual of Clinical Microbiology. 9th ed. Washington DC.: Asm press; 2007.

4. Washington CW, Allen SD, Koneman EW, Procop GW, Janda WM, Wood GL. Koneman's color atlas and textbook of diagnostic microbiology. 6th ed. NewYork: Lippincott Williams \& Wilkins; 2009:1232-35.

5. BioMerieux India (Pvt)Ltd. BacT/ALERT manual. 2009. Ref Type: Catalog. 
6. Sharma MP, Bhatia V. Abdominal tuberculosis. Indian J Med Res 2004 Oct;120 (4):30515.

7. Piersimoni C, Scarparo C, Callegaro A, Tosi CP, Nista D, Bornigia S, et al. Comparison of $\mathrm{MB} /$ Bact alert 3D system with radiometric BACTEC system and Lowenstein-Jensen medium for recovery and identification of mycobacteria from clinical specimens: a multicenter study. J Clin Microbiol 2001 Feb;39(2):651-7.

8. Mattei R, Savarino A, Fabbri M, Moneta S, Tortoli E. Use of the BacT/Alert MB mycobacterial blood culture system for detection of Mycobacteria in sterile body fluids other than blood. J Clin Microbiol 2009 Mar;47(3):711-4.

9. Tzoanopoulos D, Mimidis K, Giaglis S, Ritis K, Kartalis G: The usefulness of PCR amplification of the IS6110 insertion element of M. tuberculosis complex in ascitic fluid of patients with peritoneal tuberculosis. Eur J Intern Med 2003, 14(6):367-371.

10. Parija SC, Gireesh AR. Early diagnosis of tuberculous meningitis: A comparison of nested polymerase chain reaction and BacT/ALERT. Indian J Pathol Microbiol April-June 2011;(54):421-2.

11. Khan R, Abid S, Jafri W, Abbas Z, Hameed K, Ahmad Z: Diagnostic dilemma of abdominal tuberculosis in non-HIV patients: an ongoing challenge for physicians. World J Gastroenterol, 2006, 12(39):6371-5.

12. Honore-Bouakline S, Vincensini JP, Giacuzzo V, Lagrange PH, Herrmann JL. Rapid diagnosis of extrapulmonary tuberculosis by PCR: impact of sample preparation and DNA extraction. J Clin Microbiol 2003 Jun;41(6):2323-9.

13. Das S, Paramasivan CN, Lowrie DB, Prabhakar R, Narayanan PR. IS6110 restriction fragment length polymorphism typing of clinical isolates of Mycobacterium tuberculosis from patients with pulmonary tuberculosis in Madras, south India. Tuber Lung Dis 1995 Dec;76(6):550-4.

14. Sahadevan R, Narayanan S, Paramasivan CN, Prabhakar R, Narayanan PR. Restriction fragment length polymorphism typing of clinical isolates of Mycobacterium tuberculosis from patients with pulmonary tuberculosis in Madras, India, by use of direct-repeat probe. J Clin Microbiol 1995 Nov;33(11):3037-9.

15. Radhakrishnan I, MY K, Kumar RA, Mundayoor S. Implications of low frequency of IS6110 in fingerprinting field isolates of Mycobacterium tuberculosis from Kerala, India. J Clin Microbiol 2001 Apr;39(4):1683.

16. Chakravorty S, Sen MK, Tyagi JS. Diagnosis of extrapulmonary tuberculosis by smear, culture, and PCR using universal sample processing technology. J Clin Microbiol 2005 Sep;43(9):4357-62.

Table- 1 : Various clinical, radiological, cytological and histopathological findings among the cases

\begin{tabular}{|l|l|}
\hline Findings & \% of patients \\
\hline Fever & 81.5 \\
\hline Abdominal tenderness & 67.1 \\
\hline Weight loss & 74.6 \\
\hline Night sweat & 65.8 \\
\hline Abnormal peritoneal fluid cytology & 57.14 \\
\hline Raised ESR & 53.1 \\
\hline
\end{tabular}

Journal of Evolution of Medical and Dental Sciences/Volume1/Issue5/November-2012Page-759 
ORIGINAL ARTICLE

\begin{tabular}{|l|l|}
\hline Positive Mantoux test & 100 \\
\hline Caeseating granuloma in histopathology & 14.2 \\
\hline Past history of pulmonary tuberculosis & 21.4 \\
\hline
\end{tabular}

Table - 2: Microbiological findings among various subgroups of tuberculous peritonitis (TBP)

\begin{tabular}{|c|c|c|c|c|c|c|c|}
\hline \multirow[b]{2}{*}{ Sub groups } & \multirow[b]{2}{*}{$\begin{array}{l}\text { No of } \\
\text { cases }\end{array}$} & \multicolumn{6}{|c|}{ Number of cases positive for M. tuberculosis by } \\
\hline & & Zn staining & $\begin{array}{l}\text { AP } \\
\text { staining }\end{array}$ & $\begin{array}{l}\text { LJ } \\
\text { culture }\end{array}$ & $\begin{array}{l}\text { BacT/ } \\
\text { ALERT }\end{array}$ & $\begin{array}{l}\text { Nested } \\
\text { PCR }\end{array}$ & $\begin{array}{l}\text { Combined } \\
\text { BacT/ALERT \& } \\
\text { Nested PCR } \\
\end{array}$ \\
\hline Total cases & 21 & 05 & 06 & 12 & 16 & 19 & 20 \\
\hline $\begin{array}{c}\text { Confirmed } \\
\text { TBP }\end{array}$ & 07 & 03 & 03 & 07 & 07 & 07 & 07 \\
\hline $\begin{array}{c}\text { Probable } \\
\text { TBP }\end{array}$ & 14 & 02 & 03 & 05 & 09 & 12 & 13 \\
\hline $\begin{array}{l}\text { Control(Non } \\
\text { TBP) }\end{array}$ & 09 & 0 & 0 & 0 & 0 & 01 & 0 \\
\hline
\end{tabular}

Figure-1: Gel image after nested PCR targeting IS6110 of M.tuberculosis

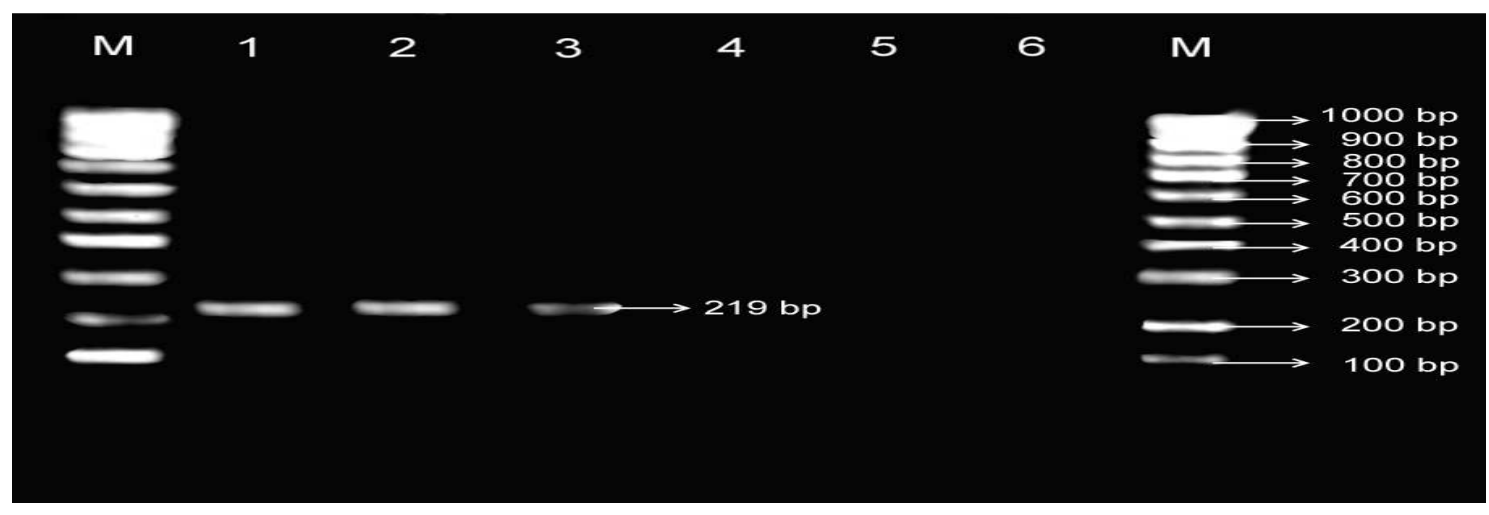

Lane M : 100bp DNA ladder, Lane 2 : 219bp PCR product of positive control,

Lane2\&3: 219bp PCR product of clinical control,

Lane 4 to 6 : Clinical samples negative for IS6110 of M.tuberculosis 